\title{
DISRUPÇÃO INSTITUCIONAL, EMPREENDEDORISMO E PLATAFORMAS TECNOLÓGICAS: MECANISMOS DE DISRUPÇÃO SOB VIÉS DA ABORDAGEM PRÁTICA
}

Wilquer Ferreira ${ }^{1}$

${ }^{1}$ Pontifícia Universidade Católica de Minas Gerais - PUC Minas 


\section{DISRUPÇÃO INSTITUCIONAL, EMPREENDEDORISMO E PLATAFORMAS TECNOLÓGICAS: MECANISMOS DE DISRUPÇÃO SOB VIÉS DA ABORDAGEM PRÁTICA}

Resumo: As transformações promovidas pelos avanços tecnológicos, sobretudo pelas plataformas tecnológicas de consumo compartilhado têm impactado drasticamente diversos setores e gerado alterações no ambiente institucional. Apesar da existência de vários estudos voltados para as instituições e seus processos de mudança, sobretudo no campo da prática, poucos abordam o processo de disrupção institucional, isto é, quando uma lógica institucional dominante é substituida por outra, com novas crenças, regras, normas, ideologias e sistemas regulatórios. O presente artigo aborda esse tipo particular, embora negligenciado, tipo de disrupção, através da perspectiva institucional prática. Constata-se que as plataformas reúnem mecanismos que podem influenciar as ações e interações dos atores institucionais, que ao modificar as práticas institucionalizadas, podem interromper (disrupt) instituições, resultando em uma lógica suprimida e outra dominante, contexto onde está inserida a ação empreendedora. Observa-se que a disrupção institucional, apesar de pouco explorada, constitui um fator inerente às plataformas de consumo compartilhado.

Palavras-chave: Disrupção Institucional. Abordagem prática. Plataforma tecnológica. Empreendedorismo.

As novas tecnologias têm promovido uma série de perturbações no ambiente institucional, alterando a forma como as pessoas se relacionam, utilizam os recursos disponíveis, desempenham as atividades cotidianas e formulam "as regras e exigências às quais as organizações individuais devem se conformar para receber legitimidade e apoio" (Scott, 1995, p.132).

Essa ruptura das instituições pode ser demonstrada com o caso do setor de mobilidade urbana, onde empresas de compartilhamento de carros e de caronas, como Drivy e a Uber, demonstram estar prejudicando a propriedade de carros particulares e os sistemas de transporte (Martin \& Shaheen, 2011; Meyer \& Shaheen, 2017).

Ao contrário das organizações tradicionais, as plataformas de compartilhamento operam localmente, em nível da cidade, e, portanto, também estão sujeitas às pressões institucionais exclusivas da cidade em que operam, além das regras e restrições nacionais (Zvolska, Palgan, \& Mont, 2019). Além dessas organizações serem influenciadas por seus ambientes institucionais, elas também podem moldar, construir ou interromper (disrupt) instituições (Thornton, Ocasio, \& Lounsbury, 2012) introduzindo uma lógica de plataforma (Cannon \& Summers, 2014; Laurell \& Sandström, 2016; Mair \& Reischauer, 2017; Geissinger, Laurell, \& Sandstrom, 2018).

Alguns pesquisadores têm abordado o tema da ruptura institucional como produto das plataformas tecnológicas. Laurell e Sandström (2016) demonstram, em um estudo de caso da Uber, que as plataformas de economia compartilhada são percebidas como uma ruptura institucional porque conseguiram difundir os mercados estabelecidos. Outros autores também discutiram a ruptura de mercado (Cohen \& Sundararajan, 2015; Martin, Upham, \& Budd, 2015; 
Wosskow, 2014), bem como a interrupção do emprego causada pelas plataformas de compartilhamento urbano (Drahokoupil \& Fabo, 2016; Sundararajan, 2016). A ruptura foi creditada a uma variedade de fatores tecnológicos e socioeconômicos, como a ascensão das TICs (Tecnologias da Informação e Comunicação), a utilização de smartphones, e as crises econômicas, que levaram as pessoas a aceitarem novas formas de emprego e renda (Murillo, Buckland, \& Val, 2017).

Zvolska, Palgan e Mont (2019) argumentam que a ascensão da economia compartilhada interrompe não apenas os mercados e as relações de emprego, mas também as práticas aceitas associadas à tradicional redistribuição de recursos. Neste contexto diversos atores institucionais seriam afetados, incluindo usuários, empreendedores, planejadores, formuladores de políticas públicas e acadêmicos (Meyer \& Shaheen, 2017).

A medida que as plataformas ganham força, processos de turbulência institucional e competitiva podem ser invocados em mais setores da economia, impactando diversos segmentos (Geissinger, Laurell, \& Sandstrom, 2018), sendo disruptivas para as instituições vigentes, e criando novas instituições (Lawrence \& Suddaby, 2006).

As teorias institucionais tem assumido que as instituições possuem uma lógica central (Friedland \& Alford,1991), ou racionalidade (DiMaggio \& Powell, 1983; Scott, 1995, 2001; Townley, 2002), dotadas de um conjunto de práticas materiais e simbólicas, além de princípios organizacionais que fornecem lógicas de ação para indivíduos e organizações (Glynn \& Lounsburry, 2005; Suddaby \& Greenwood, 2005).

Alguns estudos examinam como uma lógica institucional é substituída por uma nova lógica (ex. Cooper, Hinings, Greenwood \& Brown, 1996; Zilber, 2006), que resultam em uma lógica suprimida e outra lógica dominante (Ex. Reay \& Hinnings, 2005; Townley, 2002), que podem ser tecnologias desconectadas de pressupostos morais, ou normas particulares com enfraquecimento de pressupostos consolidados. Entretanto, apesar de sua relevância, a disrupção institucional, seus mecanismos e consequências, têm sido negligenciada por teóricos organizacionais (Zvolska, Palgan, \& Mont, 2019; Denis, Langley \& Rouleau, 2007; Kraatz \& Block, 2007), especialmente em estudos sobre plataformas tecnológicas (Zervas, Proserpio, \& Byes, 2017; Zvolska, Palgan, \& Mont, 2019; Laurell \& Sandstrom, 2016; Christensen, McDonald, Altman, Elizabeth, \& Palmer, 2016).

Diante do exposto, o presente artigo, um ensaio teórico, tem como questão de pesquisa: Como a abordagem institucional prática pode auxiliar no estudo dos mecanismos promotores de disrupção institucional em plataformas tecnológicas?

Ao buscar respostas para esse problema, o presente trabalho procura avançar no estudo das plataformas tecnológicas através de três mecanismos promotores de disrupção institucional sob viés da abordagem prática: a) abandono de sanções e recompensas; b) dissociação de fundamentos morais, regras e tecnologias institucionalizadas; e c) supressão de suposiçõos e crenças.

O objetivo central é investigar a temática da disrupção institucional em plataformas tecnológicas, partindo do resgate dos principais mecanismos que levam à ruptura e a mudança institucional através da perspectiva prática. De forma a desenvolver a temática, o trabalho está estruturado em três partes: a primeira resgata a literatura sobre teoria institucional e suas vertentes; na segunda, aborda-se os processos de mudança institucional e os mecanismos geradores de turbulência institucional através da perspectiva institucional prática. Já na terceira 
parte, apresenta-se um quadro analítico sobre os mecanismos promotores de disrupção institucional, contexto o qual insere-se as plataformas de compartilhamento. Finalmente, nas considerações finais, as reflexões são resgatadas e organizadas, apresentando a disrupção institucional como um processo resultante da invocação de novos "vocabulários de prática", que sinalizam que novas identidades estão surgindo, onde novos objetivos, novas práticas, e novos interesses são internalizados.

\section{Teoria Institucional: Contribuições da Perspectiva Prática}

Uma instituição é definida por seus componentes duros, que podem ser descritos como as regras do jogo (North, 1990) ou esquemas, com componentes leves que sustentam os componentes duros (Clemens \& Cook, 1999). Há interdependência entre as instituições e os sistemas nos quais elas estão inseridas (Hira \& Hira, 2000; Peters, 2005; Pierson, 2004).

Alguns estudiosos argumentam que as instituições praticamente determinam as ações dos indivíduos (Friel, 2017) a medida que definem as preferências e o poder na sociedade (Powell \& DiMaggio, 1991; Thelen \& Steinmo, 1992), e ao mesmo tempo em que fornecem os significados compartilhados e quadros cognitivos que moldam como os humanos interpretam o comportamento dos outros (Fligstein, 2001; Hall \& Taylor, 2003).

A teoria institucional tem evoluido ao longo dos últimos anos "sob o arcabouço dual do funcionalismo e da ciência normal" (Clegg \& Hardy, 2006, p. 30), sendo abrangida por teóricos de diversos campos do conhecimento, como a sociologia (Dimaggio \& Powell, 1988; Roy, 1997), a teoria organizacional (Meyer \& Rowan, 1977), as ciências políticas (Bonchek \& Shepsle, 1996), as ciências econômicas (North, 1990), entre outras.

A teoria institucional pode ser abordada através de várias vertentes, três delas tradicionalmente conhecidas como: a escolha racional, o institucionalismo histórico e a vertente sociológica; além dessas, ainda existem a vertente discursiva abordada por Schmidt (2010), e duas vertentes neoinstitucionalistas: o institucionalismo construtivista e o institucionalismo das redes (Hall \& Taylor, 2003). Ambas as perspectivas não são excludentes, mas complementares entre si (Lima, Machado, \& Gerassi, 2011; Friel, 2017; Schmidt; 2010; Hall \& Taylor, 2003). A perspectiva da escolha racional pressupõe que os atores tenham preferências fixas e agem racionalmente para maximizar suas preferências. Nessa perspectiva as instituições buscam reduzir incertezas (Théret, 2003), e influenciam os atores à medida que incentivam suas ações. Os teóricos dessa escola muitas vezes se baseiam em explicações funcionalistas para a existência das instituições (Friel, 2017).

O institucionalismo histórico concebe as instituições como conjuntos de práticas regularizadas (Schmidt, 2010). As instituições fornecem modelos racionais e morais que levam os indivíduos a agirem (Hall \& Taylor, 2003, p.198), em um processo "path dependency", onde assume-se a existência de uma causalidade social dependente da trajetória percorrida (Collier \& Collier, 1991)

A abordagem sociológica examina como os atores seguem regras e normas, assumindo que identidades e culturas são as fontes de interesses para os indivíduos. A ação humana é examinada em termos de seus propósitos simbólicos e cerimoniais não utilitários (Schmidt, 2010). Essa abordagem enfatiza como as instituições moldam os atores e não como os atores formam as instituições. A capacidade de decisão racional é afetada pelas contingências do 
ambiente, que por sua vez é composto por valores, crenças e regras criadas a partir de uma interação social (Dimaggio \& Powell, 1983; Meyer \& Rowan, 1977). Os neoinstitucionalistas dessa corrente concebem as instituições de forma mais global, incluindo não só as regras, procedimentos e normas formais, mas também os sistemas de símbolos, esquemas cognitivos e os modelos morais que guiam a ação humana (Castro, 2013).

O institucionalismo construtivista compreende a mudança institucional como parte da relação entre os atores e o contexto em que estão inseridos, enquanto desdobramento dessas interações. Essa corrente focaliza as mudanças complexas que acontece na instituição após a sua constituição (Lima, Machado, \& Gerassi, 2011).

O institucionalismo de redes compreende as redes enquanto instituições, na medida em que representam padrões estáveis ou recorrentes de interações e trocas entre indivíduos, grupos e organizações (Lima, Machado, \& Gerassi, 2011). Assim, os teóricos dessa corrente vêem as redes como variáveis que afetam a distribuição do poder, a construção de interesses e identidades, além das dinâmicas que caracterizam essas interações (Castro, 2013).

Finalmente, o institucionalismo discursivo examina como os atores geram e legitimam ideias por meio de uma lógica de comunicação, focalizando o processo interativo através do qual as ideias são geradas. As instituições fornecem a estrutura para o discurso, definindo a gama de ideias que são mais ou menos aceitáveis nas interações discursivas (Schmidt, 2010). Schmidt (2010) pondera que as várias vertentes do institucionalismo devem ser consideradas como complementares entre si, ao invés de diferentes formas de compreender os fenômenos.

A vertente institucional sociológica admite em suas ramificações neoinstitucionais a abordagem institucional prática. Essa abordagem examina como atores interagem com os construtos e recorrem às ferramentas sociais e físicas no contexto de suas atividades rotineiras, que constituem o campo da prática (Lawrence \& Suddaby, 2006).

Os teóricos da prática (ex. Bourdieu, 1990; Giddens, 1984; Sztompka, 1991; Turner, 1994) aderem a dualidade entre instituições e prática, ou seja, como as instituições são construídas pela, e ao mesmo tempo, também constroem a ação (Jarzabkowski, Matthiesen, \& Van de Ven, 2009). Enquanto neoinstitucionalistas focam nas mudanças institucionais atribuindo maior importância na agência (ex. Jepperson, 1991; Oliver, 1991; Seo \& Creed, 2002), os teóricos da prática atribuem foco na ação, interações e negociações entre múltiplos atores (Jarzabkowski, Balogun, \& Seidl, 2007). Nessas ações e interações os atores iniciam, reproduzem e modificam as práticas institucionalizadas através de hábitos, conhecimento tácito, cultura, rotinas, motivações e emoções (Reckwitz, 2002).

A abordagem prática focaliza nas ações e interações entre atores para criar, manter e interromper (disrupt) instituições (Jarzabkowski, Matthiesen, \& Van de Ven, 2009). Isto é, as práticas diárias dos atores produzem instituições pluralísticas e as interações são repletas de tensões institucionais, ao invés de uma fenômeno excepcional ou isolado. Para esse fluxo de interações que ocorrem na estrutura institucional, Sztompka (1991, p. 96) propõe o conceito de práxis, constituído por um processo social de constante mutação, onde as organizações e seus atores constroem e reconstroem suas logícas institucionais através de práticas diárias, imersas em interações.

Thornton, Ocasio e Lounsbury (2012) argumentam que as práticas e suas identidades estão no centro analítico das lógicas institucionais, respondendo por mudanças endógenas em 
um campo institucional, e fornecendo as bases de identidade e mobilização coletiva, onde as variações da prática respondem pela transformação institucional.

As lógicas institucionais movem-se através da linguagem - através da teoria e do esquema, da estrutura e da narrativa - o que "constitui mutuamente as representações simbólicas das lógicas institucionais e suas práticas materiais" (Thornton, Ocasio, \& Lounsbury, 2012, pp. 149-150). Thornton, Ocasio e Lounsbury (2012) explicam que as narrativas, em particular, são centrais, pois são "relatos explícitos de práticas concretas e eventos moldados em interação, fornecendo um elo crucial entre práticas materiais e construções simbólicas" (p. 156). Esquemas tornam-se quadros que são combinados em narrativas que "geram ligações específicas entre os elementos simbólicos e materiais da lógica institucional" (p. 159). Teorias, molduras e narrativas, "embutidas em vocabulários de prática", são mídias do movimento institucional. As narrativas "levam à formação de vocabulários de prática" e podem, ao vincular categorias às práticas, fazer emergir novas lógicas institucionais (Thornton, Ocasio, \& Lounsbury, 2012, pp. 159-160).

Diante de um quadro amplo e variado de vertentes que se constitui a teoria institucional, aborda-se aqui especificamente o tema da inovação, mudança e ruptura institucional através da abordagem institucional prática, que oferece a bagagem conceitual para tratar o tema de mudança e disrupção institucional.

\section{Inovação, Mudança e Ruptura Institucional}

Existem vários desafios de moldar novas instituições, lidando com a inércia institucional (Chen, 2008), e transformando as instituições existentes para que funcionem de forma mais eficaz (Amable, 2000). As questões tornam-se cada vez mais prementes no contexto da mudança global para modelos de desenvolvimento baseados em inovação, onde fortes sistemas científicos e tecnológicos influenciam a vantagem competitiva das economias nacionais (Archibugi \& Pietrobelli, 2003; Fagerberg, Srholec, \& Knell, 2007; Fagerberg \& Srholec, 2008; Porter, 1998).

Compreender a mudança institucional também tem sido importante para o estudo da inovação (Hage \& Meeus, 2009; Hollingsworth, 2000). A análise institucional nas frentes de pesquisas sobre inovação tem se baseado cada vez mais no conceito de sistemas nacionais de inovação (Edquist \& Johnson, 2000; Lundvall, Vang, Joseph, \& Chaminade, 2009), enraizado em uma perspectiva evolucionista sobre mudança institucional (Nelson \& Winter, 1982). Os sistemas nacionais de inovação são compostos por instituições, formas organizacionais e interações entre eles (Etzkowitz \& Leydesdorff, 2000).

A mudança institucional depende do contexto, que pode ser cultural, ambiental ou estrutural. É essa relatividade que dificulta o significado da institucionalização, porque a propriedade pode mudar. A melhor maneira se pensar a institucionalização é vê-la como um estado particular de uma instituição (Brady, 2001).

A teoria das instituições investiga as origens das convenções e de outros comportamentos institucionalizados, valendo-se particularmente da economia dos custos de transação e da teoria dos jogos. A mudança institucional tem uma analogia com a mudança tecnológica, sendo caracterizada pela dependência de trajetória e possibilidades de múltiplos equilíbrios (McNicoll, 2001). 
A estrutura institucional de uma sociedade é historicamente contingente e, portanto, em alguma medida acidental. A mudança institucional, assim como a mudança na tecnologia, depende do caminho (a direção da mudança é restringida não apenas pelo seu estado atual, mas também pela sua história), isso porque os resíduos da ação social passada, limitam as possibilidades da ação social subsequente. Entretanto, a mudança também é influenciada por esperanças e expectativas sobre o futuro, que não são apenas reflexos das opções imediatamente percebidas, mas são formadas a partir de informações sobre o mundo e as oportunidades que ele parece apresentar (McNicoll, 2001).

Voss (2015) pondera que existem muitos tipos diferentes de processos propensos a gerar ou alterar instituições sociais. Entre dois extremos há o mecanismo de mudança por design consciente e o mecanismo de mudança por forças evolutivas. No primeiro, instituições formais, como as normas legais, são, em muitos casos, planejadas propositadamente, tais como as comissões parlamentares nas democracias modernas que propõem regularmente tais mudanças, sendo normalmente acompanhadas ou complementadas por mudanças de instituições informais na sociedade em geral. Essas regras informais geralmente mudam de forma mais incremental. O segundo mecanismo envolve as forças evolutivas de uma "mão invisível" (Adam Smith). Nesse caso, as ações orientadas por objetivos de um grande número de atores podem produzir resultados que não parecem intencionais, devido ao planejamento consciente de uma autoridade central. Mas, na verdade, a instituição é um subproduto da atividade de cada indivíduo para se adaptar localmente às suas circunstâncias. Instituições desse tipo são chamadas de "ordens espontâneas", que uma vez adotadas, podem ser incluídas em uma tradição cultural e serão ensinadas às crianças por seus pais (por exemplo, modos à mesa) (Sugden, 1986; Young, 1998).

A maioria das instituições empiricamente observáveis não pode ser explicável exclusivamente por um desses dois tipos de mecanismos extremos ou ideais, mas provavelmente são combinações complexas de diferentes tipos de processos (Voss, 2015). A mudança institucional tende a ser lenta e imponente, mas às vezes rompe definiticamente com o passado, ou responde rapidamente à circunstâncias que mudam rapidamente. Novas instituições são inventadas, outras desaparecem; e outras devem se adaptar para se manterem sustentáveis (Harries, 2012).

Harries (2012) argumenta que a mudança institucional tende a vir de quatro fontes principais: a) empreendedores institucionais - as últimas décadas presenciaram a nomeação de executivos do setor privado como chefes de agências governamentais, com a missão explícita de introduzir novas práticas e culturas de gestão; b) sobreposição estrutural entre organizações participantes - a fronteira entre os setores público e privado está cada vez mais difusa, trazendo novos participantes com diferentes origens e interesses; c) choques externos e internos do ambiente de trabalho tais como mudanças radicais na economia ou na tecnologia - novas tecnologias de rede fornecem novas capacidades e oportunidades para as instituições, alterando fundamentalmente os fluxos de informação e comunicação; d) lógicas institucionais concorrentes que guiam a escolha e a tomada de decisão - mudanças nas práticas, crenças e valores subjacentes que moldam o comportamento e as ações resultantes das mudanças sociais e econômicas na sociedade como um todo (Harries, 2012).

As teorias sobre mudança institucional tipicamente distinguem entre mudanças lentas e disruptivas. As causas para ambas podem ser exógenas ou endógenas. Os choques exôgenos podem ocorrer devido à fatores externos como guerras, mudanças climáticas, políticas, etc. Em 
uma mudança disruptiva nas instituições causada por mudanças endógenas, o equilíbrio institucional, isto é, a compatibilidade, entre as regras formais, regras informais e a ideologia formal dominante subjacente é quebrada, por exemplo, por grandes inovações técnicas ou organizacionais (Sauerland, 2015), que ocorrem no interior dos sistemas produtivos (Schumpeter, 1942). Isso ocorre pelo fato dos indivíduos, com o tempo, adaptarem seus comportamentos ao conjunto existente de regras institucionais, investindo na aprendizagem e na construção de padrões bem-sucedidos de comportamento. Esse processo resulta em uma dependência de trajetória do sistema institucional (Sauerland, 2015).

Para que haja uma mudança institucional lenta bem-sucedida, as novas instituições formais devem ser complementares às instituições informais existentes e à ideologia formal dominante (North, 1991). Em contraste, as mudanças disruptivas são tipicamente causadas por choques exógenos, ou a nível endógeno, pela inserção de tecnologias capazes de alterar drasticamente o paradigma vigente (Sauerland, 2015).

Neste contexto, a disrupção institucional ocorre quando uma lógica dominante é substituída por outra (novas crenças, regras, normas e sistemas regulatórios), convivendo, porém, esta com outras lógicas institucionais múltiplas. O processo de disrupção ocorre em conjunto com a criação e a manutenção, onde os atores tentam discreditar o modelo lógico institucional anterior, ao mesmo tempo em que procuram introduzir e promover o recém-criado, além de desenvolver maneiras para difundir e manter seus modelos preferidos. Esta co-criação institucional ocorre simultaneamente com a disrupção, bem como o desenvolvimento de mecanismos de manutenção destinados a sustentar a lógica institucional em um processo contínuo (Lawrence, Suddaby, \& Leca, 2009).

O pluralismo institucional tem sido utilizado para explicar as variações na difusão das práticas institucionais, onde diferentes lógicas possibilitam alternativas viáveis em empresas dentro de um mesma indústria (Lounsbury, 2007), outros estudos examinam o pluralismo institucional como um conjunto de mudanças institucionais, onde uma lógica institucional é substituída por uma nova lógica (ex. Cooper, Hinings, Greenwood, \& Brown, 1996; Zilber, 2006), que resultam em uma lógica suprimida e outra lógica dominante (Reay \& Hinnings, 2005; Townley, 2002).

Lawrence e Suddaby (2006) distinguem três tipos de mecanismos de dirupção institucional: a) abandono de sanções e recompensas; b) dissociação de fundamentos morais, regras e tecnologias institucionalizadas; e c) supressão de suposições e crenças.

$\mathrm{O}$ abondono de sanções e recompensas refere-se à redefinição de conceitos e ideias bem estabelecidas através da ação coercitiva de atores poderosos que poderiam levar a uma mudança institucional revolucionária. Esse tipo de trabalho institucional ocorre por meio do judiciário, que permite que os atores estatais e não-estatais removam diretamente recompensas e sanções de práticas, tecnologias e regras institucionalizadas. Os atores também podem perturbar as instituições indiretamente "minando as definições técnicas e as suposições nas quais foram fundadas" (Lawrence \& Suddaby, 2006, p. 236).

A dissociação de fundamentos morais gradualmente perturba os fundamentos normativos das práticas, regras ou tecnologias institucionalizadas. Nesse processo as fundações normativas são mais comumente interrompidas por atores da elite e poderosos, mas suas atividades geralmente não visam diretamente atacá-los (Lawrence \& Suddaby, 2006). 
A supressão de suposições e crenças ocorre quando os atores removem alguns dos custos de transação associados às práticas, tecnologias e regras predominantes, garantindo assim a inovação e reduzindo os riscos associados à diferenciação. Os atores podem minar premissas e crenças, criando uma inovação que rompa as configurações institucionais existentes ou minando gradualmente as instituições por meio de práticas contrárias (Lawrence \& Suddaby, 2006).

Zvolska, Palgan e Mont (2019) argumentam que os atores perturbam as instituições quando a ordem institucional existente não fornece apoio suficiente para que eles realizem suas atividades. Frequentemente, os atores que trabalham para criar novas instituições podem inadvertidamente romper as regras, práticas e tecnologias existentes (Lawrence \& Suddaby, 2006), de modo que a criação institucional está fortemente ligada à ruptura institucional.

Uma vez descritos os mecanismos que podem levar a disrupção institucional a partir da abordagem institucional prática, torna-se relevante analisar as plataformas tecnologícas com base nos mecanismos de disrupção institucional (Lawrence \& Suddaby; 2006).

\section{Plataformas Tecnológicas e Disrupção Institucional}

Em relação ao abandono de sanções e recompensas, as plataformas tecnológicas de compartilhamento são propensas a modificar as estruturas institucionais por meio do trabalho regulatório se seus objetivos estiverem alinhados com as instituições normativas e sociocognitivas existentes (Zvolska, Palgan, \& Mont, 2019). A Uber, por exemplo, abriu uma perspectiva empreendedora para indivíduos, ao exigir apenas o cadastro de motoristas na plataforma como prestadores de serviços em tempo parcial. Não sendo necessário todos os requisitos normativos exigidos para os taxistas. Esse fato gerou diversas reivindicações, a exemplo das corporações de taxistas, que clamam por restrições e a regulamentação dos serviços.

A dissociação de fundamentos morais gradualmente perturba os fundamentos normativos das práticas, regras ou tecnologias institucionalizadas (Lawrence \& Suddaby; 2006). As plataformas de compartilhamento urbano estão questionando a instituição da propriedade como um pré-requisito para um modo adequado de consumo. A maioria das plataformas de compartilhamento baseiam seus modelos de negócios em minar a necessidade de propriedade, através do fornecimento do acesso a bens, serviços e habilidades. Para tanto, trabalham para desinstitucionalizar a propriedade em bases morais, promovendo a utilização de recursos como alternativa de consumo mais sustentável.

As plataformas também estão indiretamente prejudicando as instituições normativas predominantes, incentivando e possibilitando conjuntos de práticas que minam as fundações das instituições estabelecidas, tais como a promoção da ideia do consumo sem dono, do compartilhamento e do consumo de bens de "segunda mão". Essas concepções, anteriormente pouco admitidas socialmente, atualmente estão enquadrados no estilo de vida moderno (Zvolska, Palgan, \& Mont, 2019). O consumo de um bem baseado em acesso, diferentemente do consumo baseado em propriedade, possui o benefício de liberar o indivíduo de quaisquer obrigações de natureza econômica, social ou emocional, advindas com a propriedade desse bem (Botsman \& Rogers, 2010). Essa condição afeta atitudes e comportamentos de consumo e 
desafia pressupostos e padrões sociais fortemente enraizados (Zervas, Proserpio, \& Byes, 2017).

Ao oferecer aos usuários benefícios de uso a custos menores, sendo uma alternativa à propriedade tradicional (Botsman \& Rogers, 2010), as plataformas provocam uma ruptura com a noção de valorização da propriedade privada, um dos fundamentos do mundo capitalista (Zvolska, Palgan, \& Mont, 2019), e sugerem a emergência de elementos eventualmente deflagradores de um novo tipo de "paradigma técnico econômico", ou ciclo sociotécnico (Kondratieff, 1982).

A supressão de suposições e crenças ocorre quando os atores removem alguns dos custos de transação associados às práticas, tecnologias e regras predominantes, garantindo assim a inovação e reduzindo os riscos associados à diferenciação (Lawrence \& Suddaby; 2006). Os atores/empreendedores podem minar premissas e crenças, criando uma inovação que rompa as configurações institucionais existentes, ou minando gradualmente as instituições por meio de práticas contrárias. Uma inovação apoiada por tecnologias de mecanismos de referência e feedback, em plataformas de compartilhamento urbano, ajuda a substituir modelos comportamentais existentes e facilita novas maneiras de criar confiança entre estranhos.

No caso da Uber e de tantas outras plataformas de serviços, que baseiam suas experiências em sistemas "peer-to-peer" (que substitui o modelo tradicional "business-toperson"), a confiança é construída através de um sistema de reputação baseada em transparência, confiança e legitimidade (Perren \& Kozinets, 2018). A maioria investe na criação de sistemas de avaliação e classificação, alimentados pelos próprios usuários individualmente (fornecedores e/ou clientes), úteis tanto para o aperfeiçoamento do próprio sistema, como, também, para subsidiar processos decisórios de usuários efetivos e, também, potenciais. É assim que todo usuário da Uber é, sistematicamente avaliado, seja como demandante (cliente avaliado pelo motorista), seja como ofertante ou fornecedor do serviço (motorista avaliado pelo consumidor). A tecnologia está minando suposições culturais cognitivas sobre hospedar estranhos em casa ou compartilhar pertences com estranhos. Reduz os riscos associados à nova prática e reduz os custos de transação ao empregar soluções tecnológicas. Outra suposição que é prejudicada por essa inovação é o típico papel de policiamento do Estado; em plataformas online, um novo sistema de policiamento e revisão por pares é utilizado (Zvolska, Palgan, \& Mont, 2019).

Nesse contexto, as plataformas de compartilhamento reuniriam mecanismos promotores de disrupção institucional (Zvolska, Palgan, \& Mont, 2019), seja por meio do trabalho regulatório (abandono de sanções e recompensas); trabalhando para desinstitucionalizar a propriedade em bases morais, promovendo a utilização de recursos em marcha lenta como alternativas de consumo mais sustentáveis (dissociação de fundamentos morais, regras e tecnologias institucionalizadas); ou minando suposições culturais cognitivas tais como hospedar estranhos em casa ou compartilhar pertences com estranhos, a exemplo da AirbnB e Uber, que apoiadas por tecnologias com mecanismos de referência e feedback substituem modelos comportamentais existentes, gerando confiança entre estranhos e reduzindo custos de transação.

A Tabela 1 apresenta uma síntese dos mecanismos de disrupção institucional pela perspectiva prática e as características das plataformas de compartilhamento que indicariam o caráter disruptivo institucional desse tipo de plataforma, em especial da Uber. 
Tabela 1

Disrupção Institucional e Plataformas de Compartilhamento

\begin{tabular}{l|l|}
\hline \multicolumn{1}{c|}{$\begin{array}{c}\text { Mecanismos de } \\
\text { Disrupção }\end{array}$} & Disrupção Institucional \\
\hline $\begin{array}{l}\text { Abandono de sanções } \\
\text { / recompensas }\end{array}$ & $\begin{array}{l}\text { Definição de conceitos e } \\
\text { ideias bem estabelecidos } \\
\text { através da ação coercitiva de } \\
\text { atores poderosos que } \\
\text { poderiam levar a uma } \\
\text { mudança institucional } \\
\text { "revolucionária" (Lawrence } \\
\text { \& Suddaby, 2006, p. 236). }\end{array}$ \\
\hline
\end{tabular}

Plataformas Tecnológicas de Compartilhamento / UBER

- São propensas a modificar as estruturas institucionais por meio de trabalho regulatório removendo diretamente recompensas e sanções, de práticas, tecnologias e regras institucionalizadas (Zvolska, Palgan, \& Mont, 2019) afetando atitudes e comportamentos de consumo e desafiando pressupostos e padrões sociais fortemente enraizados (Zervas, Proserpio, \& Byes, 2017). - Uber apenas exige o cadastro de motoristas na plataforma como fornecedores/ prestadores de serviços em tempo parcial (UBER, 2019). Não sendo necessário todos os requisitos normativos exigidos para os taxistas.

\begin{tabular}{ll|lll}
\hline Dissociação de & Perturba os fundamentos
\end{tabular} fundamentos morais, normativos das práticas, regras e tecnologias regras ou tecnologias institucionalizadas institucionalizadas

(Lawrence \& Suddaby, 2006; Harries, 2012).
Supressão suposições e crenças.
Atores removem alguns dos custos de transação associados às práticas, tecnologias e regras predominantes, rompendo as configurações institucionais existentes ou minando gradualmente as instituições por meio de práticas contrárias (Lawrence \& Suddaby, 2006; Voss 2015).
- Questionam a instituição da propriedade como um pré-requisito para um modo adequado de consumo. Enfatizam as práticas de compartilhamento com estranhos e consumo de bens de segunda mão com viés de sustentabilidade (Zvolska, Palgan, \& Mont, 2019). - A Uber promove a ideia do motorista é quem manda no seu tempo, sem chefe e com horário de trabalho flexível (UBER,2019).

- A Uber estimula o abandono da propriedade veicular e o consumo do serviço oferecido pela plataforma (UBER, 2019).

-A prática de dar feedback aos pares em plataformas online está mudando gradualmente as suposições das pessoas sobre fazer negócios com estranhos. Novas instituições cognitivas estão sendo formadas e normalizadas, e as pessoas estão gradualmente aceitando essas práticas (Zvolska, Palgan, \& Mont, 2019).

-Na Uber a confiança é construída através de um sistema de reputação baseada em transparência, confiança e legitimidade (Perren \& Kozinets, 2018), substituindo modelos comportamentais existentes, gerando confiança entre estranhos e reduzindo custos de transação.

Nota. Fonte: Elaboração Própria.

As plataformas tecnológicas de consumo compartilhado, ao minar o valor de propriedade do bem, promoveriam impactos diretos na lógica institucional, visto que o valor é central para uma lógica institucional, sendo a fonte da legitimidade de suas regras, uma base de identificação individual para o arbítrio, e o alicerce sobre o qual os seus poderes são constituídos (Zvolska, Palgan, \& Mont, 2019). As lógicas institucionais são sustentadas não apenas por práticas materiais modais, mas por identificações pessoais com um valor institucional (Thornton, Ocasio, \& Lounsbury, 2012), as plataformas ao introduzirem um novo conceito de 
valor, alteram a forma como as pessoas se identificam com os valores institucionais, o que levaria a dirupção institucional.

\section{Considerações Finais}

Retomando o objetivo de investigar como abordagem prática pode auxiliar no estudo dos mecanismos promotores de disrupção institucional em plataformas tecnológicas, observase que a abordagem institucional prática torna-se útil no entendimento da disrupção institucional em plataformas, dado que essas reúnem mecanismos que podem influenciar as ações e interações dos atores/empreendedores institucionais (Slater, 2010; Weyland, 2008), que ao modificar as práticas institucionalizadas (Reckwitz, 2002; Jarzabkowski, Balogun, \& Seidl, 2007), podem criar, manter e interromper (disrupt) instituições (Jarzabkowski, Matthiesen, \& Van de Ven, 2009), resultando em uma lógica suprimida e outra dominante.

Uma nova tecnologia (McNicoll, 2001), como uma plataforma tecnológica, pode alterar a forma como os atores se identificam com um valor institucional ou um símbolo, podendo alterar as ideologias, levando à adaptação institucional para a ideologia da nova maioria (Sauerland, 2015), na qual novas crenças, regras, normas e sistemas regulatórios (Lawrence \& Suddaby; 2006) são inseridos, dando origem a novos vocabulários de prática, e em decorrência, fazendo emergir novas lógicas institucionais.

"É somente quando as teorias, molduras e narrativas estão incorporadas nas práticas que as ideias se tornam lógicas" (Thornton, Ocasio \& Lounsbury, 2012, p. 162). Neste sentido, quando alterações nas representações simbólicas (teorias, molduras e narrativas), são incorporadas nas práticas, novas lógicas institucionais emergem, o que implica que novos valores serão internalizados. Desta forma, a invocação de novos "vocabulários de prática", sinalizam que novas identidades estão surgindo com novos objetivos, novas práticas e novos interesses (Thornton, Ocasio, \& Lounsbury, 2012) e, portanto, levariam a disrupção institucional, resultando em uma lógica suprimida e outra lógica dominante (Lawrence \& Suddaby; 2006).

Entre os mecanismos promotores de disrupção institucional em plataformas, aqui foram destacados: a) o abandono de sanções, recompensas e tecnologias, onde os atores redefinem conceitos e ideias bem estabelecidos; b) dissociação de fundamentos morais, regras e tecnologias institucionalizadas, na qual as fundações normativas são mais comumente interrompidas por atores da elite e poderosos; e c) supressão de suposições e crenças, onde uma inovação rompe drasticamente as configurações institucionais existentes, ou de forma gradual, através de práticas contrárias (Lawrence \& Suddaby; 2006). Esses mecanismos representam avanços no entendimento da disrupção institucional em plataformas, e abrem oportunidades de investigação de outros mecanismos de disrupção institucional a serem explorados.

A disrupção institucional constitui um campo frutífero de oportunidades de pesquisas. Uma delas poderia analisar as várias dimensões e caminhos pelos quais as lógicas institucionais, identidades coletivas e práticas emergem e mudam com o tempo. Outra poderia investigar como as lógicas institucionais são alteradas a partir da inserção de novas tecnologias, frutos da ação empreendedora, tais como as plataformas de compartilhamento, contribuindo sobretudo no aspecto da inovação, já que a compreensão da mudança institucional também tem sido importante para o estudo da inovação (Hage \& Meeus, 2009, Hollingsworth, 2000). Outra 
ainda, poderia evocar os processos de mudança nos vocabulários de prática e como os membros organizacionais percebem as mudanças e criam sentido (sensemaking), e também transmitem sentido (sensegiving). Contexto onde está inserido o intraempreendedor.

Espera-se que as reflexões aqui inseridas possam estimular a realização de pesquisas empíricas sobre o fenômeno de disrupção institucional em plataformas, bem como um melhor entendimento dos impactos e mecanismos deflagradores desse processo.

\section{Referências}

Amable, B. (2000). Institutional complementarity and diversity of social systems of innovation and production. Review of International Political Economy, 7, 645-687.

Archibugi, D., \& Pietrobelli, C. (2003). The globalisation of technology and its implications for developing countries - windows of opportunity or further burden? Technol. Forecast. Soc. Chang, 70, 861-883.

Bonchek, M.S., \& Shepsle, K.A. (1996). Analyzing politics: Rationality, behavior and instititutions. New York:W.W. Norton \& Co.

Botsman, R., \& Rogers, R. (2010). Beyond Zipcar: Collaborative Consumption. Harvard Business Review, 88(October), 30.

Bourdieu, P. (1990). The logic of practice. Cambridge, UK: Polity Press.

Brady, D. W. (2001). Institutionalization. In: Smelser, Neil J. and Baltes, Paul B. (Eds.). International Encyclopedia of the Social and Behavioral Sciences, 11, Oxford: Elsevier Science Ltd, pp. 7.558-7.561.

Cannon, S., \& Summers, L.H. (2014). How Uber and the sharing economy can win over regulators. Harvard Bussiness Review, 13 (10), 24-28.

Castro, L. M. A. (2013). Do neoinstitucionalismo tradicional às redes: as mudanças colocadas a partir do modelo de governança. In: XXXVII Encontro Nacional da ANPAD, Rio de Janeiro. Anais do XXXVII Encontro da ANPAD. Rio de Janeiro.

Collier, D., \& Collier, R. (1991). Shaping the Political Arena. Princeton: Princeton University Press.

Cohen, M., \& Sundararajan, A. (2015). Self-Regulation and Innovation in the Peer-ToPeer Sharing Economy. University of Chicago Law Review Dialogue, 82, 116-133.

Cooper D. J., Hinings B., Greenwood R., \& Brown, J. L. (1996). Sedimentation and transformation in organizational change: The case of Canadian law firms. Organization Studies, 17, 623-647.

Chen, L., 2008. Institutional inertia, adjustment, and change: Japan as a case of a coordinated market economy. Rev. Int. Polit. Econ, 15, 460-479.

Clegg, S. R., \& Hardy, C. (2006). Handbook de estudos organizacionais: modelos de análise e novas questões em estudos organizacionais. 3a. ed. São Paulo: Atlas.

Clemens, E.S., \& Cook, J.M. (1999). Politics and institutionalism: explaining durability and change. Annu. Rev. Sociol. 25, 441-466.

Christensen C, McDonald R, Altman, Elizabeth J., \& Palmer J. (2016). Disruptive Innovation: Intellectual History and Future Paths. Boston: Harvard Business School Press.

Denis, J-L, Langley, A; \& Rouleau, L. (2007). Strategizing in pluralistic contexts: Rethinking theoretical frames. Human Relations, 60(1); 179-215. 
DiMaggio, P. J., \& Powell, W. W. (1983). The iron cage revisited: Institutional isomorphism and collective rationality in organizational fields. American Sociological Review, 48, 147160.

Dimaggio, P. J. (1988). Interest and agency in institutional theory. In L. G. Zucker. Institutional patterns and organizations. Cambridge, MA: Ballinger.

Drahokoupil, J., \& Fabo, B. (2016). The platform economy and the disruption of the employment relationship. In: European Economic, Employment and Social Policy, vol. 5.

Edquist, C., \& Johnson, B. (2000). Institutions and organisations in systems of innovation. In: Edquist, C., McKelvey, M. (Eds.), Systems of Innovation: Growth, Competitiveness and Employment. Edward Elgar, Cheltenham, UK, pp. 42-61.

Etzkowitz, H., \& Leydesdorff, L. (2000). The dynamics of innovation: from National Systems and 'Mode 2' to a triple helix of university-industry-government relations. Res. Policy, 29, 109-123.

Fagerberg, J., Srholec, M., \& Knell, M. (2007). The competitiveness of nations: Why some countries prosper while others fall behind. World Dev. 35, 1595-1620.

Fagerberg, J., Srholec, M. (2008). National innovation systems, capabilities and economic development. Res. Policy 37, 1417-1435.

Friedland, R. \& Alford, R. R. (1991). Bringing society back in: Symbols, practices, and institutional contradictions. In W. W. Powell \& P. J. DiMaggio (eds.), The new institutionalism in organizational analysis, Chicago: University of Chicago Press, pp. 232263.

Friel, D. (2017). Understanding institutions: different paradigms, different conclusions. Revista de Administração, 52, 212-214.

Fligstein, N. (2001). Social Skill and the Theory of Fields. Sociological Theory, 19(2), 105125.

Geissinger, A., Laurell, C., \& Sandström, C. (2018). Beyond uber and airbnb-tracking thelong tail of the sharing economy. Technol. Forecast. Soc. Chang. Ahead of print: https://doi.org/10.1016/j.techfore.2018.06.012(in press)

Giddens, A. (1984). The Constitution of Society. Cambridge, UK: Polity Press.

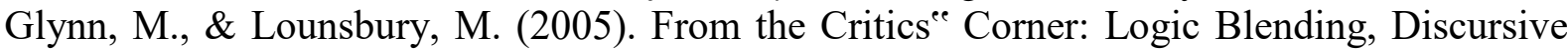
Change and Authenticity in a Cultural Production System. Journal of Management Studies, 42(5), 1031-1055.

Hage, J., \& Meeus, M. (2009). Innovation, Science, and Institutional Change: A Research Handbook. Oxford University Press, New York.

Hall, P. A., \& Taylor, R. C. R. (2003). As três versões do neo-institucionalismo. Lua Nova, São Paulo.

Harries, S. (2012). Innovation and change: ideas, networks and communities. Editor(s): Stephen Harries, In Chandos Information Professional Series, Records Management and Knowledge Mobilisation, Chandos Publishing, pp. 115-141.

Hira, A., \& Hira, R. (2000). The new institutionalism: contradictory notions of change. Am. J. Econ. Sociol, 59, 267-282.

Hollingsworth, J.R., (2000). Doing institutional analysis: implications for the study of innovations. Rev. Int. Polit. Econ. 7, 595-644. 
Jarzabkowski, P., Balogun, J, \& Seidl, D. (2007). "Strategizing: The challenges of a practice perspective ${ }^{e}$. Human Relations, 60(1), 5-27.

Jarzabkowski, P., Matthiesen, J., \& Van de Vem, A. (2009). Doing which work? A practice approach to institutional pluralismo. In Lawrence T., Leca, B. and R. Suddaby. (Eds) Institutional Work: Actors and Agency in Institutional Studies of Organizations. Cambridge, UK: Cambridge University Press.

Jepperson, R. L. (1991). Institutions, institutional effects, and institutionalism. In W. W. Powell \& P. J. DiMaggio (Eds.) The new institutionalism in organizational analysis. Chicago: University of Chicago Press, pp. 143-163.

Kondratieff N. D. (1982). The Long Waves in Economic Life. The Review of Economics and Statistics. The MIT Press, 17, 6 (Nov., 1935), 105-115.

Kraatz, M., \& Block, E. (2007). Organizational Implications of Institutional Pluralism". Forthcoming in The Handbook of Organizational Institutionalism. University of Illinois, Champaign, Ilinois. Retrieved from https://www.profemilyblock.com/uploads/3/2/0/2/32024349/kraatz_and_block__institutional_pluralism.pdf

Laurell, C., \& Sandstrom, C., 2016. Analysing uber in social media - disruptive tech- $€$ nology or institutional disruption? Int. J. Innov. Manag., 20 (5), 1-19.

Lawrence, T.B., \& Suddaby, R., 2006. Institutions and institutional work. In: Clegg, S.R.H., Cynthia, Lawrence, Tom, Nord, Walter R. (Eds.), The SAGE Handbook of Organization Studies, second ed. SAGE Publications Inc, London, pp. 215-254.

Lawrence, T.B., Suddaby, R., \& Leca, B., 2009. Introduction: theorizing and studying institutional work. In: Leca, B., Suddaby, R., Lawrence, T.B. (Eds.), Institutional Work: Actors and Agency in Institutional Studies of Organizations. Cambridge University Press, Cambridge, pp. 1-28.

Lawrence, T., Suddaby, R., \& Leca, B. (2011). Institutional Work: Refocusing Institutional Studies of Organization. Journal of Management Inquiry, 20(1), 52-58.

Lima, L.D., Machado, C.V., \& Gerassi, C.D. (2011). O neo-institucionalismo e a análise de políticas de saúde: contribuições para uma reflexão crítica. In MATTOS, R. A.; BAPTISTA, T. W. F. Caminhos para análise das políticas de saúde, 1, 111-137.

Lounsbury, M. (2007). A tale of two cities: Competing logics and practice variation in the professionalizing of mutual funds. Academy of Management Journal, 50(2), 280-307.

Lundvall, B.-A., Vang, J., Joseph, K.J., \& Chaminade, C. (2009). Innovation system research and developing countries. In: Lundvall, B.-A., Joseph, K.J., Chaminade, C., Vang, J. (Eds.), Handbook of Innovation Systems and Developing Countries: Building Domestic Capabilities in a Global Setting. Edward Elgar, Northampton, MA, pp. 1-30.

Mair, J., \& Reischauer, G. (2017). Capturing the dynamics of the sharing economy: institutional research on the plural forms and practices of sharing economy organizations. Technol. Forecast. Soc. Chang, 125, 11-20.

Martin, C.J., Upham, P., \& Budd, L. (2015). Commercial orientation in grassroots social innovation: insights from the sharing economy. Ecol. Econ.,118, 240-251.

Martin, E., \& Shaheen, S. (2011). The impact of carsharing on public transit and nonmotorized travel: an exploration of North American carsharing survey data. Energies, 4(11), 20942114 
Meyer, J., \& Rowan, B. (1977). Institutionalized organizations: formal structure as myth and ceremony. American Journal of Sociology, 83(2), 340-363.

Meyer, G., \& Shaheen, S. (2017). Disrupting Mobility: Impacts of Sharing Economy and Innovative Transportation on Cities. Springer, pp. 1-349.

McNicoll, G. (2001). Fertility: Institutional and Political Approaches. Editor(s): Neil J. Smelser, Paul B. Baltes, International Encyclopedia of the Social \& Behavioral Sciences, Pergamon, pp. 5538-5543.

Murillo, D., Buckland, H., \& Val, E. (2017). When the sharing economy becomes neoliberalism on steroids: unravelling the controversies. Technol. Forecast. Soc. Change 125, 66.

Nelson, R., \& Winter, S. (1982). An Evolutionary Theory of Economic Change. Belknap Press, Cambridge, MA.

North, D.C. (1990). Institutions, Institutional Change and Economic Performance. Cambridge University Press, Cambridge.

North, D.C. (1991). Institutions. Journal of Economic Perspectives, Pittsburgh (USA), 5(1), 97 112.

Oliver, C. (1991). Strategic responses to institutional processes. Academy of Management Review, 16, 145-179.

Peters, B.G. (2005). Institutional Theory in Political Science: The New Institutionalism. Continuum, London.

Perren, R., \& Kozinets, R. V. (2018). Lateral Exchange Markets: How Social Platforms Operate in a Networked Economy. Journal of Marketing, 82(1), 20-36.

Pierson, P. (2004). Politics in Time: History, Institutions, and Social Analysis. Princeton University Press, Princeton, NJ.

Porter, M.E. (1998). Clusters and the new economics of competition. Harv. Bus. Ver, 76, 7790.

Powell, W.W., \& DiMaggio, P. (1991). The New Institutionalism in Organizational Analysis.

University of Chicago Press, Chicago.

Reay, T., \& Hinings, C. R. (2005). The recomposition of an organizational field: Health care in Alberta. Organization Studies, 26(3), 351-384.

Reckwitz, A. (2002). Towards a theory of social practice: A development in cultural theorizing. European Journal of Social Theory, 5(2), 243-63.

Roy, W. G. (1997). Socializing capital: The rise of the large industrial corporation in America. Princeton University Press, Princeton, New Jersey.

Sauerland, Dirk. (2015). Ideologies, Institutions, and the New Institutionalism, Editor(s): James D. Wright, International Encyclopedia of the Social \& Behavioral Sciences (Second Edition), Elsevier, 2015, pp. 561-572.

Scott, W. R. (1995). Institutions and Organizations: Theory and Research. Sage Publications, London;

Scott, W. R. (2001). Institutions and organizations, 2nd Edition. Thousand Oaks, CA: Sage.

Schmidt, V. (2010). Taking ideas and discourse seriously: Explaining change through discursive institutionalism as the fourth 'new institutionalism'. European Political Science Review, 2(1), 1-25.

Schumpeter, J.A. (1942). Capitalism, Socialism, and Democracy. New York: Harper and Brothers. 
Seo, M., \& Creed, W. E. D. (2002). Institutional contradictions, praxis, and institutional change: A dialectical perspective. Academy of Management Review, 27, 222-247.

Suddaby, R., \& Greenwood, R. (2005). Rhetorical strategies of legitimacy. Administrative Science Quarterly, 50, 35-67.

Sugden, Robert. (1986). The Economics of Rights, Cooperation and Welfare. Oxford: Basil Blackwell.

Sundararajan, A. (2016). The Sharing Economy: the End of Employment and the Rise of CrowdBased Capitalism. MIT Press, Cambridge.

Slater, D. (2010). Altering authoritarianism: institutional complexity and autocratic agency in Indonesia. In: Mahoney, J., Thlen, K. (Eds.), Explaining Institutional Change. Cambridge University Press, New York, pp. 132-167.

Sztompka, P. (1991). Society in action: The theory of social becoming. Cambridge, UK: Polity Press

Thelen K, \& Steinmo S. (1992). Historical institutionalism in comparative politics. In: Thelen $\mathrm{K}$, Steinmo S, Longstreth F, orgs. Structuring Politics: historical institutionalism en comparative analysis. Cambridge: Cambridge University Press, pp. 1-32.

Théret B. (2003). As instituições entre as estruturas e as ações. Lua Nova, 58, 225-255.

Thornton, P.H., Ocasio, W., \& Lounsbury, M. (2012). The Institutional Logics Perspective: a New Approach to Culture, Structure and Process. Oxford University Press, Oxford.

Townley, B. (2002). The role of competing rationalities in institutional change. Academy of Management Journal, 45, 163-179.

Turner, S. (1994). The Social Theory of Practices. Cambridge, UK: Polity Press.

Uber. (2019). Encontrando o caminho Criando possibilidades para usuários, motoristas e cidades. Retirado em Janeiro 10, 2019, de https://www.uber.com/pt-BR/our-story/.

Voss, Thomas R. (2015). Institutions. Editor(s): James D. Wright, International Encyclopedia of the Social \& Behavioral Sciences (Second Edition). Elsevier, pp. 190-195.

Weyland, K. (2008). Toward a new theory of institutional change. World Polit. 60, 281-314.

Wosskow, D. (2014). Unlocking the Sharing Economy: an Independent Review. Department for Business, Innovation and Skills, United Kingdom.

Young, H. Peyton. (1998). Individual Strategy and Social Structure. Princeton NJ:Princeton University Press.

Zervas, G., Proserpio, D., \& Byers, J. W. (2017). The Rise of the Sharing Economy: Estimating the Impact of Airbnb on the Hotel Industry. Journal of Marketing Research, 54(5), 687705 .

Zilber, Tamar, B. (2006). The Work of theSymbolic in Institutional Processes: Translationsof Rational Myths in Israeli High Tech, Academy of Management Journal, 49(2), 281-303.

Zvolska L., Palgan Y. V., \& Mont O. (2019). How do sharing organisations create and disrupt institutions? Towards a framework for institutional work in the sharing economy, Journal of Cleaner Production, 219, 667-676. 